\title{
Utilización del ácido tranexámico diluido en la solución anestésica local
}

\author{
Use of the tranexamic acid diluted in the local anesthetic solution
}

\author{
Guillermo Blugerman', Diego Schavelzon'1, Isaac Zilinsky², Giuseppe Del Torto³, \\ Miguel Mussi Becker ${ }^{1}$, Victoria Schavelzon', Guido Blugerman ${ }^{1}$
}

REVISTA ARGENTINA DE CIRUGÍA PLÁSTICA 2019;25(1):8-10. DOI/10.32825/RACP/201901/0008-0010

\section{INTRODUCCIÓN}

Desde su desarrollo en los años 50', el ácido tranexámico (ATX) ha demostrado eficiencia comprobada en la prevención del sangrado y resangrado intra- y posoperatorio por su efecto antifibrinolítico así como su actividad antiinflamatoria en procedimientos quirúrgicos en una amplia gama de especialidades médicas. El trauma ha sido un campo de investigación muy fértil, en el que se han destacado grandes ventajas de su uso en los casos agudos evitando un gran número de muertes.

Muchas especialidades quirúrgicas, como la cirugía cardiaca y la ortopédica, han reducido la pérdida de sangre y la posterior necesidad de transfusiones ${ }^{1}$. También es utilizado en eventos hemorrágicos por aumento de la fibrinólisis, como en la epistaxis, la hemoptisis y la hemorragia gastrointestinal ${ }^{2}$. Dentro del campo de la ginecología se utiliza la vía oral para disminuir el sangrado y el dolor de la menstruación, y es una buena opción para la menorragia ${ }^{3}$. Se utiliza de manera efectiva para controlar el sangrado en el embarazo, ya que el ATX puede pasar a través de la placenta ${ }^{4}$.

Las distintas formas de administración utilizadas han sido la intravenosa, la oral y la tópica. Muy poco se ha investigado sobre su uso como un componente agregado en la solución anestésica local o la anestesia tumescente. A pesar de los beneficios mencionados, la cirugía plástica ha empezado a investigar su uso en cirugías reparadoras y maxilofaciales en primera instancia, y en forma tardía en la cirugía estética, probablemen-

1. Centro Médico B\&S de Excelencia en Cirugía Plástica, Buenos Aires, Argentina

2. Department of Plastic and Reconstructive Surgery, Sheba Medical Center, Tel Hashomer, affiliated to Sackler School of Medicine, Tel Aviv University, Tel Aviv, Israel

3. Università degli studi della Campania "Luigi Vanvitelli", Servicio de Cirugía Plástica, Napoli, Italia

$\triangle$ Correspondencia: sacper@sacper.org.ar

Los autores no declaran conflictos de intereses te porque no involucra cirugías con riesgo de sangrado masivo que puedan requerir transfusiones como en el caso de las cirugías ortopédicas o cardíacas. Sin embargo, existen otros beneficios que aporta el ATX, como la reducción de la equimosis y la inflamación, que son de gran importancia en los tiempos actuales donde la rápida recuperación y sin secuelas cosméticas es muy deseada.

Un problema adicional de sangrado intra- y posoperatorio lo constituyen los depósitos de hemosiderina que muchas veces quedan como pigmentos instalados en las capas profundas de la dermis. Estas manchas son muy difíciles de tratar, por lo que la prevención de esta complicación es esencial. Esto lo podemos lograr mediante la utilización del ATX.

En este trabajo exponemos nuestra experiencia utilizando el ATX diluido en la solución anestésica local y en la solución tumescente para su uso en los diferentes procedimientos de la cirugía plástica estética.

\section{ÁCIDO TRANEXÁMICO}

Patentado en el año 1957, el ATX es un análogo sintético de la lisina que inhibe competitivamente la activación del plasminógeno en plasmina, evitando temporalmente de esta manera la disolución y degradación de los coágulos de fibrina ${ }^{6}$. Es así como adquiere la capacidad de disminuir el sangrado intra- y perioperatorio al alterar selectivamente el sistema fibrinolítico. Además, el ATX bloquea la activación plaquetaria inducida por la plasmina preservando así las plaquetas para la posterior formación del coágulo ${ }^{6}$.

Al inhibir la formación de la plasmina, el ATX también aporta un efecto antiinflamatorio debido a que la plasmina es responsable de varias actividades inflamatorias ${ }^{7}$.

Las dosis terapéuticas in vitro requirieron aproximadamente $10 \mu \mathrm{g} / \mathrm{ml}$ para conseguir una inhibición del 80\% de la activación del plasminógeno ${ }^{8}$. Esto se traduce a una dosis intravenosa de $10 \mathrm{mg} / \mathrm{kg}$ con un efecto terapéutico de hasta por lo menos 17 horas en los tejidos ${ }^{8}$. 
TABLA 1. Lista de procedimientos quirúrgicos.

\begin{tabular}{|l|c|}
\hline Cirugías & $N^{\circ}$ pacientes \\
\hline $\begin{array}{l}\text { Cirugías faciales: ritidectomía, lifting de cejas, } \\
\text { otoplastia, blefaroplastia, queiloplastia, bichectomía. }\end{array}$ & 333 \\
\hline $\begin{array}{l}\text { Contorno corporal: abdominoplastia, flancoplastia, } \\
\text { gluteoplastia, braquioplastia, musloplastia, bodylift. }\end{array}$ & 210 \\
\hline Mamas: aumento, reducción, pexia, ginecomastia. & 341 \\
\hline Rinoplastia. & 111 \\
\hline Lipoaspiración. & 1081 \\
\hline Microtransplante capilar. & 18 \\
\hline Miscelaneas. & 277 \\
\hline Total & 2371 \\
\hline
\end{tabular}

Las contraindicaciones de su uso para un tratamiento terapéutico incluyen comorbilidades como insuficiencia renal, enfermedades neoplásicas, cardiovasculares, respiratorias, tratamiento antiagregante previo y antecedentes de enfermedades tromboembólicas como trombosis venosa profunda (TVP), accidente cerebrovascular $(\mathrm{ACV})$ y hemorragia subaracnoidea ${ }^{9,10}$. Se deberán tener en cuenta otros factores de riesgo de un posible evento tromboembólico, tales como el embarazo, tratamiento con anticonceptivos orales, tabaquismo o viajes de larga distancia. Los pacientes deben ser evaluados con mucho cuidado mediante una exhaustiva historia clínica que permita identificar estas comorbilidades.

Si bien en la actualidad la utilización del ATX ha ganado terreno dentro del campo de la cirugía plástica mediante su administración intravenosa, oral y/o tópica, para encontrar un antecedente de su utilización diluido en la anestesia local o tumescente nos tenemos que referir al trabajo del cirujano iraní $\mathrm{Namazi}^{11}$, quien en el año 2007 publica por primera vez sobre la posibilidad de la utilización del ATX en la solución anestésica local (solución de Namazi) para lograr disminuir el sangrado en las cirugías de microtrasplante capilar. El mismo autor, en el año 2014, propone su aplicación para la reducción de la equimosis e inflamación en el posoperatorio de la lipoaspiración, en una breve comunicacion ${ }^{12}$.

En noviembre de 2017, el Dr. Isaac Zilinsky nos realizó una comunicación verbal de sus trabajos y su experiencia con el uso del ATX disuelto en la solución anestésica local, la cual utilizaba en sus pacientes operados de cáncer de piel mediante la técnica de Mohs13. Ya había publicado algunas comunicaciones preliminares sobre la infiltración subcutánea de solución anestésica local con ATX en las blefaroplastias superiore ${ }^{14}$. Finalmente, en 2019 se publicó su trabajo sobre las cirugías cutáneas para resección de carcinomas de piel mediante técnica de Mohs con resultados altamente satisfactorios, logrando demostrar la reducción del sangrado aun en pacientes anticoagulados 15 .

\section{MATERIALES Y MÉTODOS}

A partir de la comunicación verbal que recibimos del Dr. Zilinsky, iniciamos el uso del ATX desde mediados de noviembre de 2017 hasta febrero de 2019. Fueron realizados en total 2371 procedimientos de cirugía plástica
TABLA 2. Solución anestésica local.

\begin{tabular}{|l|c|}
\hline Solución salina $0,9 \%$ & $20 \mathrm{ml}$ \\
\hline Lidocaína & $400 \mathrm{mg}$ \\
\hline Adrenalina & $1 \mathrm{ml} / 1 \mathrm{mg}$ \\
\hline Ácido tranexámico & $500 \mathrm{mg}$ \\
\hline
\end{tabular}

estética (Tabla 1), 1921 (81\%) en mujeres y 450 (19\%) en hombres; la edad media fue de $36 \pm 5$ años.

Hemos protocolizado la utilización del ATX basados en la dosis máxima de $10 \mathrm{mg} / \mathrm{kg}$ aceptada por la bibliografía para la vía endovenosa. En la solución anestésica local (Tabla 2) utilizamos $500 \mathrm{mg}$ de ATX, la cual laaplicamosen las rinoplastias, blefaroplastias, otoplastias y la extracción de las bolas de Bichat. Debido a que el ATX presenta un $\mathrm{pH}$ alcalino hemos dejado de utilizar el bicarbonato en la anestesia local.

En todas las demás cirugías llevadas a cabo hemos utilizado solución tumescente, ocupando una dosis máxima de 1 gramo distribuidos en el número total de litros utilizados (Tabla 3).

A excepción de las cirugías de contorno corporal más extensas, en las cuales se utilizó anestesia general con infiltración de solución anestesia local (9\%), todas las demás cirugías fueron realizadas con solución tumescente asociada a sedación endovenosa (91\%) realizada por el anestesiólogo. En los pacientes bajo anestesia general, se redujo la dosis de lidocaína a la mitad en cada litro de solución tumescente. Se excluyeron pacientes con antecedentes de enfermedad tromboembólica y neoplásica.

\section{RESULTADOS}

Hemos observado la disminución del sangrado en el tiempo intraoperatorio evaluando el número de compresas utilizadas y notando la reducción en la utilización del electrocauterio durante estas cirugías. También notamos una marcada disminución en la formación posoperatoria de edema, equimosis y hematomas en comparación a nuestra práctica previa a la utilización del ATX, y una disminución en el sangrado que mojaba los apósitos absorbentes colocados sobre las heridas quirúrgicas. Identificamos solo un caso de hematoma de la hemicara derecha posterior a un lifting facial en un paciente de sexo masculino que no respetó el reposo posoperatorio y bebió alcohol, el cual fue resuelto dentro de las 24 hs de la cirugía sin mayores complicaciones. El 100\% de los pacientes fueron dados de alta en el mismo día de la intervención. Ninguno de los pacientes presentó efectos adversos ni reacciones sistémicas asociadas al ATX.

\section{DISCUSIÓN}

Aunque la eficacia del ATX ha sido ampliamente demostrada, algunos estudios han cuestionado su seguridad en relación con una mayor incidencia de eventos tromboembólicos ${ }^{16,17}$ (trombosis venosa profunda, em- 
TABLA 3. Solución anestésica tumescente.

\begin{tabular}{|c|c|c|c|c|}
\hline & Facial & Cirugía de contorno & Lipoaspiración & Mamas \\
\hline Solución salina 0,9\% & $500 \mathrm{ml}$ & $\begin{array}{c}\text { Dependiente de la extensión } \\
\text { del procedimiento }\end{array}$ & $\begin{array}{c}\text { Dependiente de la extensión } \\
\text { del procedimiento }\end{array}$ & $1000 \mathrm{ml}$ \\
\hline Lidocaína & $600 \mathrm{mg}$ & $600 \mathrm{mg}$ & $600 \mathrm{mg}$ & $1200 \mathrm{mg}$ \\
\hline Adrenalina & $1 \mathrm{ml} / 1 \mathrm{mg}$ & $1 \mathrm{ml} / 1 \mathrm{mg}$ & $15 \mathrm{ml}$ & $2 \mathrm{ml} / 2 \mathrm{mg}$ \\
\hline $\mathrm{NaHCO}_{3}$ al $8,4 \%$ & $15 \mathrm{ml}$ & $15 \mathrm{ml}$ & $\begin{array}{c}1 \mathrm{~g} \text { distribuido en el número } \\
\text { de litros utilizados }\end{array}$ & $30 \mathrm{ml}$ \\
\hline Ácido tranexámico & $500 \mathrm{mg}$ & $\begin{array}{c}1 \mathrm{~g} \text { distribuido en el número } \\
\text { de litros utilizados }\end{array}$ & \multicolumn{2}{c|}{$500 \mathrm{mg}$} \\
\hline
\end{tabular}

bolia pulmonar, infarto agudo de miocardio, accidente cerebrovascular). Sin embargo, Myles et al. ${ }^{18}$ han publicado recientemente los resultados del estudio ATACAS (Aspirin and Tranexamic Acid for Coronary Artery Surgery), un ensayo clínico multicéntrico, randomizado y cegado que involucróa 4631 pacientes sometidos a cirugía coronaria, que confirma que el ATX no aumenta el riesgo de eventos tromboembólicos.

Si bien el ATX es ampliamente utilizado en procedimientos quirúrgicos mayores como en cirugía ortopédica y cardíaca por lograr la reducción de la pérdida sanguínea, el beneficio principal que aporta a los procedimientos estéticos de la cirugía plástica son la reducción de complicaciones menores así como de moretones, con sus secuelas pigmentarias, y de la inflamación posoperatoria.

\section{CONCLUSIÓN}

Si bien aún la evidencia es escasa, lo cual permite una amplia posibilidad de estudio, la utilización del ATX administrado junto con la anestesia local ha demostrado, en trabajos previos del Dr. Zillinsky y cols. y en nuestra experiencia personal, ser un complemento eficaz y seguro para minimizar no solamente la pérdida de sangre perioperatoria sino también para reducir el edema y la equimosis, contribuyendo con una mejor recuperación del paciente. Se deberán realizar mayores investigaciones para establecer la dosis máxima segura en esta nueva forma de administración para poder tener más flexibilidad cuando se utiliza un mayor volumen que el que nosotros habitualmente utilizamos.

\section{BIBLIOGRAFÍA}

1. Elwatidy $S$, Jamjoom Z, Elgamal E, et al. Efficacy and safety of prophylactic large dose of tranexamic acid in spine surgery: a prospective, randomized, double-blind, placebo-controlled study. Spine 2008;33:2577-2580.

2. White A, O Reilly BF. Oral tranexamic acid in the management of epistaxis. Clin Otolaryngol Allied Sci 1988;13:11-16.

3. Lukes AS, Moore KA, Muse KN, et al. Tranexamic acid treatment for heavy menstrual bleeding: a randomized controlled trial. Obstet Gynecol 2010;116:865-875.

4. Kullander S, Nilsson IM. Human placental transfer of an antifibrinolytic agent (AMCA). Acta Obstet Gynecol Scand 1970;49:241-242.

5. Bala HR, Lee S, Wong C, Pandya AG, Rodrigues M. Oral Tranexamic Acid for the Treatment of Melasma: A Review. Dermatol Surg. 2018:44(6):814-825

6. $\quad \mathrm{Ng} W$, Jerath A, Wasowicz M. Tranexamic acid: A clinical review. Anaesthesiol Intensive Ther. 2015:47:339-350

7. Jimenez JJ, Iribarren JL, Lorente L, et al. Tranexamic acid attenuates inflammatory response in cardiopulmonary bypass surgery through blockade of fibrinolysis: A case control study followed by a randomized double-blind controlled trial. Crit Care 2007;11:R117

8. Andersson L, Nilsoon IM, Colleen S, Granstrand B, Melander B. Role of urokinase and tissue activator in sustaining bleeding and the management there of with EACA and AMCA. Ann N Y Acad Sci. 1968; 146:642-656.

9. Lee HC, Thng TG, Goh CL. Oral tranexamic acid (TA) in the treatment of melasma: a retrospective analysis. J Am Acad Dermatol 2016;75:385-92.
10. Anderson FA, Spencer FA. Risk factors for venous thromboembolism. Circulation 2003;107(23 Suppl 1):9-16

11. NamaziMR. Practice Pearl:A novel anesthetic solution for deacresing the blood loss at the reciepient site of hair transplatation (Namazi Solution)". Aesthetic Plast Surg 2007 Jul-Aug;31(4):415.

12. Namazi MR. Practice Pearl: Using Namazi Solution for Decreasing the Formation of Bruises in Liposuction. J Cutan Aesthet Surg. 2014 Jan-Mar;7(1):69-70

13. Zilinskyl, Barazani TB, Shenkman B, Weisman O, et al. Topical hemostatic anesthetic solution to reduce bleeding during Mohs micrographic surgery: a case control study. J Drugs Dermatol 2016;15:85-5.

14. Zilinskyl, Sagiv O, et al. Subcutaneous tranexamic acid in upper eyelid blepharoplasty: a prospective randomized pilot study". Can J Ophthalmol 2018;53(6):600-4.

15. Zilinsky I, Barazani T, Visentin D, et al. Subcutaneous Injection of Tranexamic Acid to Reduce Bleeding During Dermatologic Surgery: A Double-Blind, Placebo-Controlled, Randomized Clinical Trial. Dermatol Surg 2019 Jan 11; [EPub Ahead of Print].

16. Ker $K$, Edwards P, Perel $P$, Shakur $H$, Roberts I. Effect of tranexamic acid on surgical bleeding: Systematic review and cumulative meta-analysis. BMJ 2012:344:e3054.

17. CRASH-2 Trial Collaborators. Effects of tranexamic acid on death, vascular occlusive events, and blood transfusión in trauma patients with significant haemorrhage (CRASH-2): A randomized, placebo-controlled trial. Lancet 2010;376:23-32.

18. Myles PS, Smith JA, Painter T. Tranexamic acid in patientsundergoing coronary-artery surgery. N Engl J Med 2017;376:1893. 